\title{
Phase transition and phase diagram at a general filling in the spinless one-dimensional Holstein Model
}

\author{
Sanjoy Datta and Sudhakar Yarlagadda \\ Saha Institute of Nuclear Physics, Calcutta, India
}

(October 23, 2017)

\begin{abstract}
Among the mechanisms for lattice structural deformation, the electron-phonon interaction mediated Peierls charge-density-wave (CDW) instability in single band low-dimensional systems is perhaps the most ubiquitous. The standard mean-field picture predicts that the CDW transition occurs at all fillings and all values of the electron-phonon coupling $g$ and the adiabaticity parameter $t / \omega_{0}$. Here, we correct the mean-field expression for the Peierls instability condition by showing that the non-interacting static susceptibility, at twice the Fermi momentum, should be replaced by the dynamic one. We derive the Luttinger liquid (LL) to CDW transition condition, exact to second order in a novel blocked perturbative approach, for the spinless one-dimensional Holstein model in the adiabatic regime. The small parameter is the ratio $g \omega_{0} / t$. We present the phase diagram at non-half-filling by obtaining the surprising result that the CDW occurs in a more restrictive region of a two parameter $\left(g^{2} \omega_{0} / t\right.$ and $\left.t / \omega_{0}\right)$ space than at half-filling.
\end{abstract}

PACS numbers: 71.38.-k, 71.45.Lr, 71.30.+h, 75.10.-b

\section{INTRODUCTION}

Over the last few decades, electron-phonon interaction physics has offered a variety of intriguing and exciting phenomena such as superconductivity (inorganic and organic), CDW states, colossal magnetoresistance, metal-insulator transition, polaronic ordered phases, etc [1]. Of the electron-phonon models available, the spinless Holstein model $[2,3]$ is a simple and widely used model that mimics strongly correlated electron systems with a strong on-site coulombic repulsion and short range electron-phonon interactions. It was conjectured a long while ago by Peierls that $1 \mathrm{D}$ electron-phonon metallic systems, such as the spinless Holstein model, will undergo an electronic charge-density-wave (CDW) transition with a concomitant lattice distortion of the same periodicity [4]. Quasi-1D organic charge transfer salts [such as $\mathrm{TTF}(\mathrm{TCNQ})$ ] and conjugated polymers [such as $(\mathrm{CH})_{\mathrm{x}}$ ] as well as inorganic blue bronzes (e.g., $\mathrm{K}_{0.3} \mathrm{MoO}_{3}$ ), mixed valence Platinum chain compounds (e.g., Krogmann's salt), and transition metal chalcogenides (e.g., $\mathrm{NbSe}_{3}$ ) [5-8] exhibit such spontaneous symmetry breaking in the ground state and are good candidates for the Holstein model. Furthermore, even the strongly correlated twoband manganite systems (e.g., $\mathrm{La}_{1-\mathrm{x}} \mathrm{Ca}_{\mathrm{x}} \mathrm{MnO}_{3}$ ) [9], in the low-doped regime, can be modeled using the Holstein model $[10,11]$.

Contrary to the mean-field picture, at half-filling, it is now clear that the LL to CDW transition occurs only above a critical electron-phonon coupling strength that depends on the adiabaticity. Progress has been made over the last few decades, in terms of studying the Peierls transition at half-filling of the spinless Holstein model, by using various techniques such as quantum Monte Carlo simulations [12-15], two-cutoff renormalization- group analysis [16], variational method [17,18], densitymatrix renormalization group (DMRG) method [19], and exact diagonalization [20]. However, a controlled analytic treatment of the quantum phase transition has been reported only recently and that too only in the antiadiabatic regime [21]. Contrastingly only little effort has been devoted to understand the quantum phase transition away from half-filling [22].

The present paper is aimed at providing a well controlled analytic approach to understand the Peierls quantum phase transition in the adiabatic regime for the onedimensional spinless Holstein model at a general filling. We employ a novel blocking approach that avoids the difficulties posed by both time-dependent- and degenerateperturbation theories. Using the condition that the effective phonon frequency becomes soft at the symmetry breaking point, we obtain an instability criterion that preempts the energy levels crossing condition for phase transition. In the adiabatic regime and at halffilling, we capture the essential features of the LL-CDW transition results obtained by the "benchmark" DMRG method in Ref. [19]. At fillings other than half, we show that the LL phase certainly exists in the small polaron limit when $g^{2} \omega_{0} / t>>\max \left(1, t / \omega_{0}\right)$ and in the extreme anti-adiabatic regime. Furthermore, in the adiabatic regime, we also demonstrate that CDW phase does exist at intermediate values of the electron-phonon couplings $g<t / \omega_{0}$. We propose a qualitative phase diagram as a guide for future work.

\section{PHONON SOFTENING IN THE LL PHASE}

We begin by considering the phonon-softening in the LL phase as the signal for lattice deformation in the 1D Holstein model. The non-interacting Hamiltonian 


$$
H_{0}=\sum_{\overrightarrow{k^{\prime}}} \epsilon_{\vec{k}^{\prime}} c_{\vec{k}^{\prime}}^{\dagger} c_{\vec{k}^{\prime}}+\omega_{0} \sum_{\vec{q}} a_{\vec{q}}^{\dagger} a_{\vec{q}}
$$

and the perturbation

$$
H_{1}=\frac{g \omega_{0}}{\sqrt{N}} \sum_{\vec{q}} \rho_{\vec{q}}\left(a_{\vec{q}}+a_{-\vec{q}}^{\dagger}\right),
$$

together make up the Holstein Hamiltonian. In the above equations, $\rho_{\vec{q}}=\sum_{\vec{k}^{\prime}} c_{\vec{k}^{\prime}+\vec{q}^{\dagger}}^{\dagger} c_{\vec{k}^{\prime}}$ is the density operator, $c_{\vec{k}^{\prime}}$ is the electron destruction operator with $\vec{k}^{\prime}$ limited to the first Brillouin zone, $\epsilon_{\vec{k}^{\prime}}=-2 t \cos \left(k^{\prime}\right)$ with lattice constant being taken to be unity, $t$ is the hopping integral, $a_{\vec{q}}$ is the phonon destruction operator, $\omega_{0}$ is the optical phonon frequency, and $N$ is the number of sites. The eigen states and eigen energies of $H_{0}$ are given by $\left|\phi_{l}\right\rangle=|n ; m\rangle \equiv|n\rangle_{e l}|m\rangle_{p h}$ (with $\left|\phi_{0}\right\rangle=|0 ; 0\rangle$ being the ground state with zero phonons) and $E_{\phi_{1}}^{0}$ respectively. Whereas, for the interacting Hamiltonian $H=H_{0}+H_{1}$, the corresponding eigen states are $\left|\Phi_{l}\right\rangle$ and the eigen energies are $E_{\Phi_{l}}$.

Now the double time derivative of an operator $A$ is given by

$$
\ddot{A}=-[[A, H], H] .
$$

From the above equation, when $A$ is taken to be the ionic position coordinate $Q_{\vec{p}}=\sqrt{1 /\left(2 M \omega_{0}\right)}\left(a_{\vec{p}}+a_{-\vec{p}}^{\dagger}\right)$ and upon making the static mean-field approximation $\rho_{-\vec{p}} \propto \chi_{0}(\vec{p}, 0) Q_{\vec{p}}[8]$, we obtain the following expression:

$$
\ddot{Q}_{\vec{p}}=-\omega_{0}^{2}\left[1+2 g^{2} \omega_{0} \chi_{0}(\vec{p}, 0)\right] Q_{\vec{p}}=-\omega_{R}^{2} Q_{\vec{p}} .
$$

We know that in 1D the non-interacting polarizability $\chi_{0}(\vec{p}, 0)$ has a negative divergence at wavevector $p=2 k_{F}$. Thus it appears that the renormalized phonon frequency $\omega_{R}$ becomes soft even for vanishingly small electronphonon interaction leading to lattice distortion. We will now proceed to derive the true phonon softening condition rigorously. To this end we calculate the matrix elements of Eq. (3) and obtain

$$
\left\langle\Phi_{m}|\ddot{A}| \Phi_{n}\right\rangle=-\left(E_{\Phi_{m}}-E_{\Phi_{n}}\right)^{2}\left\langle\Phi_{m}|A| \Phi_{n}\right\rangle .
$$

When $\omega_{e}^{2}=\left(E_{\Phi_{m}}-E_{\Phi_{n}}\right)^{2} \leq 0$, instability occurs for transition from $\left|\Phi_{n}\right\rangle$ to $\left|\Phi_{m}\right\rangle$ provided that $\left\langle\Phi_{m}|A| \Phi_{n}\right\rangle \neq$ 0. For the total Hamiltonian $H_{0}+\lambda H_{1}$, we obtain $E_{\Phi_{n}}$ perturbatively to be $E_{\Phi_{n}}=E_{\phi_{n}}^{0}+\lambda^{2} E_{\phi_{n}}^{(2)}+\ldots$ where $E_{\phi_{n}}^{(2)}$ is the second order correction to the energy. Then, to second order in $\lambda$, we obtain

$\omega_{e}^{2}=\left(E_{\phi_{m}}^{0}-E_{\phi_{n}}^{0}\right)^{2}+2 \lambda^{2}\left(E_{\phi_{m}}^{0}-E_{\phi_{n}}^{0}\right)\left(E_{\phi_{m}}^{(2)}-E_{\phi_{n}}^{(2)}\right)$.

Thus to order $\lambda^{2}$ in perturbation, as the strength of the interaction is increased, $\omega_{e}^{2}=0$ before $E_{\Phi_{m}}=E_{\Phi_{n}}$.

We will now focus on the eigen states $\Phi_{l}$ and the eigen energies $E_{\Phi_{l}}$ of $H_{0}+H_{1}$ to obtain the phonon softening condition. The energy of the (expected) ground state, upto second order in perturbation, is given by:

$$
E_{\Phi_{0}}=T_{0}-\frac{g^{2} \omega_{0}^{2}}{N} \sum_{\vec{q}, m \neq 0} \frac{\left|\left\langle\left. m\right|_{e l} \rho_{\vec{q}} \mid 0\right\rangle_{e l}\right|^{2}}{\xi_{m 0}+\omega_{0}}
$$

where $T_{0}\left(=E_{\phi_{0}}^{0}\right)$ is the non-interacting kinetic energy of the $\left|\phi_{0}\right\rangle$ state and $\xi_{m 0} \equiv \xi_{m}-\xi_{0}$ with $\xi_{m}$ being the energy of $|m\rangle_{e l}$. Let $\left|\psi_{n}^{0}\right\rangle \equiv\left|0 ; n_{-\vec{p}}\right\rangle$ with $\left|n_{-\vec{p}}\right\rangle_{p h}$ corresponding to a state with $n$ phonons all of which being in the $-\vec{p}$ state. Then, the corresponding interacting state $\left|\Psi_{n}^{0}\right\rangle$ yields the energy difference $E_{\Psi_{n+1}^{0}}-E_{\Psi_{n}^{0}}=$ $\omega_{0}+\Sigma\left(-\vec{p}, \omega_{0}\right)$ where the self-energy, which is complex in general, is given by $\Sigma\left(\vec{p}, \omega_{0}\right)=g^{2} \omega_{0}^{2} \chi_{0}\left(\vec{p}, \omega_{0}\right)$ [see Appendix A for details] with the non-interacting (Lindhard) polarizability $\chi_{0}$ being defined as [23]

$$
\chi_{0}\left(\vec{p}, \omega_{0}\right) \equiv \frac{1}{N} \sum_{m \neq 0}\left[\frac{\left|\left\langle\left. m\right|_{e l} \rho_{\vec{p}} \mid 0\right\rangle_{e l}\right|^{2}}{\omega_{0}-\xi_{m 0}+i \eta}-\frac{\left|\left\langle\left. m\right|_{e l} \rho_{-\vec{p}} \mid 0\right\rangle_{e l}\right|^{2}}{\omega_{0}+\xi_{m 0}+i \eta}\right] .
$$

Hence we see that, although the above energy difference yields the expression $\omega_{e}^{2}=\omega_{0}^{2}\left[1+2 g^{2} \omega_{0} \chi_{0}\left(\vec{p}, \omega_{0}\right)\right]$ [based on Eqs. (5) and (6)] with the form being similar to that in Eq. (4), the complex nature of the self-energy complicates identifying the phonon softening condition for lattice instability [24]. We will adopt an alternate perturbative procedure to obtain the lattice instability criterion.

\section{HALF-FILLED CASE}

We begin by observing that the expression

$$
\omega_{0}=\epsilon_{\vec{k}-\vec{p}}-\epsilon_{\vec{k}}=4 t \sin \left(k_{F}-k\right) \sin \left(k_{F}\right),
$$

with $p=2 k_{F}$ has two solutions for $k$. Then at halffilling $\left(k_{F}=\pi / 2\right)$ and $k<k_{F}$, the two solutions to Eq. (8) are $\pm k=\pi / 2-\arcsin \left[\omega_{0} / 4 t\right]$. Thus, the states $\left|\psi_{1}^{1}\right\rangle \equiv|\vec{k} \rightarrow \vec{k}-\vec{p} ; 0\rangle$ (corresponding to exciting, from the ground state, the electron at $\vec{k}$ to $\vec{k}-\vec{p}$ ) and $\left|\psi_{1}^{2}\right\rangle \equiv|-\vec{k} \rightarrow-\vec{k}-\vec{p} ; 0\rangle$ are degenerate with $\left|\psi_{1}^{0}\right\rangle \equiv\left|0,1_{-\vec{p}}\right\rangle$ and are connected to $\left|\psi_{1}^{0}\right\rangle$ through $H_{1}$ as $\left\langle\psi_{1}^{1,2}\left|H_{1}\right| \psi_{1}^{0}\right\rangle \neq 0$. Hence, employing degenerate perturbation theory seems to be a natural choice to study lattice period doubling. However, the number of degenerate states that need to be considered increases linearly with number of phonons in the state $\left|\psi_{n}^{0}\right\rangle \equiv\left|0 ; n_{-\vec{p}}\right\rangle$ [see Appendix B.1]. Then, to calculate $\omega_{e}^{2}$ for large $n$ becomes difficult! 


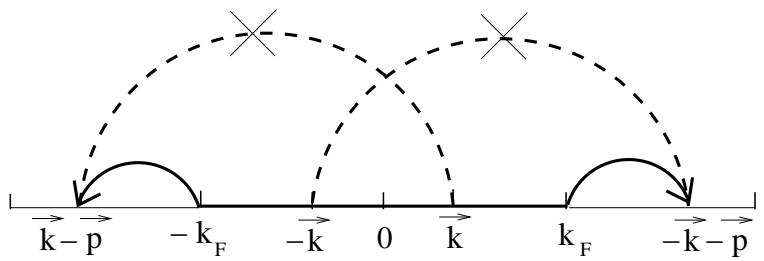

FIG. 1. Blocking procedure at half-filling. Electrons at $\mp k_{F}=\mp \pi / 2$ are excited from the ground state (dark line) to $\pm \vec{k}-\vec{p}$ to block the excitations $\pm \vec{k} \rightarrow \pm \vec{k}-\vec{p}$ respectively.

To circumvent the above problem, we adopt the following approach. We choose our starting state to be $\left|\phi_{n}\right\rangle \equiv\left|-\overrightarrow{k_{F}} \rightarrow \vec{k}-\vec{p}, \overrightarrow{k_{F}} \rightarrow-\vec{k}-\vec{p} ; n_{-\vec{p}}\right\rangle(n \geq 1)$ such that, by exciting the electrons at $\mp \vec{k}_{F}$ from the ground state to $\pm \vec{k}-\vec{p}$ outside the Fermi sea (FS), the excitations $\vec{k} \rightarrow \vec{k}-\vec{p}$ and $-\vec{k} \rightarrow-\vec{k}-\vec{p}$ are Pauli blocked [as shown in Fig. 1]. Now, $\pm \overrightarrow{k_{F}}$ have been excited for ease of mathematical manipulation. The state $\phi_{n}$, upon turning on interactions, yields the energy [see Appendix B.2 for details]:

$$
\begin{aligned}
E_{\Phi_{n}}= & E_{\Phi_{0}}+(n+1) \omega_{0}\left[1+g^{2} \omega_{0} \operatorname{Re} \chi_{0}\left(\vec{p}, \omega_{0}\right)\right] \\
& +g^{2} \omega_{0}^{2} \operatorname{Re}\left[2 \Pi_{0}\left(\vec{k}-\vec{p}, \vec{k}_{F}, \omega_{0}\right)-\chi_{0}\left(\vec{p}, \omega_{0}\right)\right],
\end{aligned}
$$

where

$$
\begin{array}{r}
\operatorname{Re} \Pi_{0}\left(\vec{s}, \vec{q}, \omega_{0}\right) \equiv-\frac{1}{N} \sum_{\vec{r}}\left[\frac{1-n_{\vec{r}}}{\epsilon_{\vec{r}}+\omega_{0}-\epsilon_{\vec{s}}}+\frac{n_{\vec{r}}}{\epsilon_{\vec{q}}+\omega_{0}-\epsilon_{\vec{r}}}\right. \\
\left.-\frac{n_{\vec{r}}}{\epsilon_{\vec{s}}+\omega_{0}-\epsilon_{\vec{r}}}-\frac{1-n_{\vec{r}}}{\epsilon_{\vec{r}}+\omega_{0}-\epsilon_{\vec{q}}}\right] .
\end{array}
$$

For $\omega_{0} /(4 t)<<1$,

$\operatorname{Re}\left[2 \Pi_{0}\left(\vec{k}-\vec{p}, \vec{k}_{F}, \omega_{0}\right)-\chi_{0}\left(\vec{p}, \omega_{0}\right)\right] \approx \frac{1}{2 \pi t} \ln \left[\frac{8 t}{9 \omega_{0}}\right]$.

The above approximation underestimates the actual value of $2 \Pi_{0}-\chi_{0}$ by less than $5 \%$ for $t / \omega_{0}>4$. In Appendix C, Fig. $6(\mathrm{c})$ shows that $2 \Pi_{0}-\chi_{0}>0$ for $t / \omega_{0}>1$. In the above Eq. (9), for $n \rightarrow \infty$, energy instability $\left(E_{\Phi_{n}}-E_{\Phi_{0}}<0\right)$ occurs for values of $g$ larger than $g_{E}$ given by $1+g_{E}^{2} \omega_{0} \operatorname{Re} \chi_{0}\left(\vec{p}, \omega_{0}\right)=0$. For $g>g_{E}, E_{n}$ has no lower bound which is an unphysical situation. Also when $g>g_{E}, E_{\Phi_{n+1}}-E_{\Phi_{n}}=1+g^{2} \omega_{0} \operatorname{Re} \chi_{0}\left(\vec{p}, \omega_{0}\right)<0$ for all $n \geq 1$ which leads to the remarkable situation that all $E_{\Phi_{n}}$ cross at the same $g=g_{E}$. To second order in the small parameter of perturbation, similar to Eq.(6), one obtains

$$
\begin{aligned}
\omega_{e}^{2}=\left(E_{\Phi_{n}}-E_{\Phi_{0}}\right)^{2} \approx & (n+1)^{2} \omega_{0}^{2}\left[1+2 g^{2} \omega_{0} \operatorname{Re} \chi_{0}\left(\vec{p}, \omega_{0}\right)\right] \\
& +2(n+1) \frac{g^{2} \omega_{0}^{3}}{2 \pi t} \ln \left[\frac{8 t}{9 \omega_{0}}\right]
\end{aligned}
$$

Thus we see from Eq. (11) that, in the adiabatic regime and for large $n$, the above mentioned energy instability occurring at $g>g_{E}$ is pre-empted by the phonon softening occurring at $g>g_{c}=g_{E} / \sqrt{2}$ with $g_{c}$ defined by the following expression:

$$
1+2 g_{c}^{2} \omega_{0} \operatorname{Re} \chi_{0}\left(\vec{p}, \omega_{0}\right)=0 .
$$

The above equation is one of our main results and is the correction to the mean-field instability condition $1+$ $2 g^{2} \omega_{0} \chi_{0}(\vec{p}, 0)=0$ obtained from Eq. (4). The operator $A$, which produces non-vanishing matrix elements in Eq. (5), is given by $A=c_{\vec{k}-\vec{p}}^{\dagger} c_{-\pi / 2} c_{-\vec{k}-\vec{p}}^{\dagger} c_{\pi / 2}\left(a_{-\vec{p}}^{\dagger}\right)^{n}$. Thus, the system becomes unstable towards absorbing a large number $(n)$ of phonons leading to a macroscopic deformation as explained below. The displacement-displacement correlation function is given by

$$
\left\langle\Phi_{n}\left|Q_{l} Q_{j}\right| \Phi_{n}\right\rangle=\left\langle\phi_{n}\left|Q_{l} Q_{j}\right| \phi_{n}\right\rangle=\frac{n \cos [(j-l) p]}{N M \omega_{0}}
$$

Thus we see that, for non-vanishing values of $n / N$, one obtains an observable ionic-position modulation when $\left|\Phi_{n}\right\rangle$ is the interacting ground state. The above Eq. (13) is true for all fillings and for any eigen state with $n_{-\vec{p}}$ phonons.

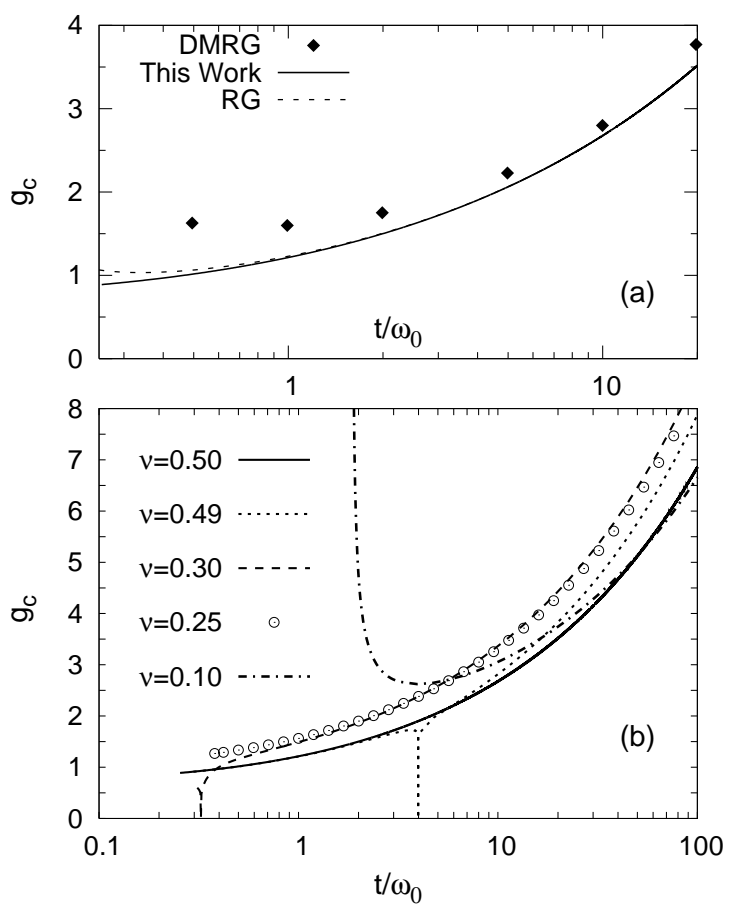

FIG. 2. Critical coupling $g_{c}$ versus adiabaticity parameter $t / \omega_{0}$. Comparison of the $g_{c}$ values obtained at (a) half-filling in this work, in Ref. [19] using DMRG, and in Ref. [16] using two-cutoff RG; and (b) various fillings $(\nu)$ in this work. 
The critical coupling $g_{c}$, given by Eq. (12), can be expressed analytically as follows:

$$
\begin{aligned}
\frac{\pi}{g_{c}^{2}}=\frac{\gamma}{\sqrt{1-\gamma^{2}}}\{\ln & {\left[\frac{\left(1-\sqrt{1-\gamma^{2}}\right)^{2}-\left(\gamma \tan k_{F}\right)^{2}}{\left(1+\sqrt{1-\gamma^{2}}\right)^{2}-\left(\gamma \tan k_{F}\right)^{2}}\right] } \\
- & \left.2 \ln \left[\frac{1-\sqrt{1-\gamma^{2}}}{1+\sqrt{1-\gamma^{2}}}\right]\right\}
\end{aligned}
$$

where $\gamma \equiv \omega_{0} /\left(4 t \sin \left(k_{F}\right)\right)<1$. Fig. 2 depicts, for various filling factors $\nu$, the variation of the critical coupling $g_{c}$ with the adiabaticity parameter $t / \omega_{0}$. At half-filling, for values of $t / \omega_{0}>5$, our theoretical curve is quite close to the numerically determined values of $g_{c}$ as reported in Ref. [19]. Furthermore, at half-filling and for $\gamma^{2}<<1$, our expression for $g_{c}$ [given by Eq. (14)] reduces to the two-cutoff renormalization result of Caron and Bourbonnais [16], i.e., $\omega_{0}=2 c t \exp \left(-\pi t / g^{2} \omega_{0}\right)$ (with $c \sim 1$ ), when we take $c=4$. The numerical agreement between the two expressions is depicted in Fig. 2(a).

\section{LESS THAN HALF-FILLING}

We will now consider fillings that are less than halffilling. The line depicted by $t / \omega_{0}=1 /\left[4 \sin \left(2 k_{F}\right) \sin \left(k_{F}\right)\right]$ [obtained by setting $k=-k_{F}$ in Eq. (8)], corresponds to the divergence of $\chi_{0}\left(\vec{p}, \omega_{0}\right)$. In the region above $t / \omega_{0}=1 /\left[4 \sin \left(2 k_{F}\right) \sin \left(k_{F}\right)\right]$ (see Fig. 3), the excitation energy expression $\epsilon_{\vec{k}-\vec{p}}-\epsilon_{\vec{k}}=\omega_{0}$ with $p=2 k_{F}$ is satisfied by one wavevector for $|k|<k_{F}$ [as seen from Eq. (8)] (see Appendix $\mathrm{C}$ for a complete analysis). We will now consider the region $t / \omega_{0}>1 /\left[4 \sin \left(2 k_{F}\right) \sin \left(k_{F}\right)\right]$. In this region, the degenerate states are $\left|\psi_{n}^{0}\right\rangle \equiv\left|0 ; n_{-\vec{p}}\right\rangle$ and $\left|\psi_{n}^{1}\right\rangle \equiv\left|\vec{k} \rightarrow \vec{k}-\vec{p} ;(n-1)_{-\vec{p}}\right\rangle$. We obtain the lower eigen energy, which corresponds to the state $\left|\psi_{n}^{-}\right\rangle \equiv$ $\left[\left|\psi_{n}^{0}\right\rangle-\left|\psi_{n}^{1}\right\rangle\right] / \sqrt{2}$, to be (see Appendix C)

$$
\begin{aligned}
E_{\Psi_{n}^{-}}= & E_{\Phi_{0}}+E^{1}+n \omega_{0}\left[1+g^{2} \omega_{0} \operatorname{Re} \chi_{0}\left(\vec{p}, \omega_{0}\right)\right] \\
& -0.5 g^{2} \omega_{0}^{2} \operatorname{Re}\left[\chi_{0}\left(\vec{p}, \omega_{0}\right)-\Pi_{0}\left(\vec{k}-\vec{p}, \vec{k}, \omega_{0}\right)\right]
\end{aligned}
$$

where $E^{1}=-\frac{\sqrt{n}}{\sqrt{N}} g \omega_{0}$ is the first order energy correction. In arriving at the above energy, we have ignored the contribution $n /\left[N\left(\epsilon_{\vec{k}-2 p}-\epsilon_{\vec{k}-\vec{p}}-\omega_{0}\right)\right]$ corresponding to exciting the electron at $\vec{k}-\vec{p}$ to the state $\vec{k}-2 \vec{p}$ by destroying a phonon of momentum $-\vec{p}$. This is valid provided $\epsilon_{\vec{k}-2 p}-\epsilon_{\vec{k}-\vec{p}}-\omega_{0} \neq 0$. The case when $\epsilon_{\vec{k}-2 p}-\epsilon_{\vec{k}-\vec{p}}-\omega_{0}=0$ (i.e., $t / \omega_{0}=1 /\left[2 \sin \left(2 k_{F}\right)\right]$ ), will be discussed in the Appendix C.1. Obviously, the macroscopic deformation instability condition is still $g>g_{c}$ with $g_{c}$ given by Eq. (12).

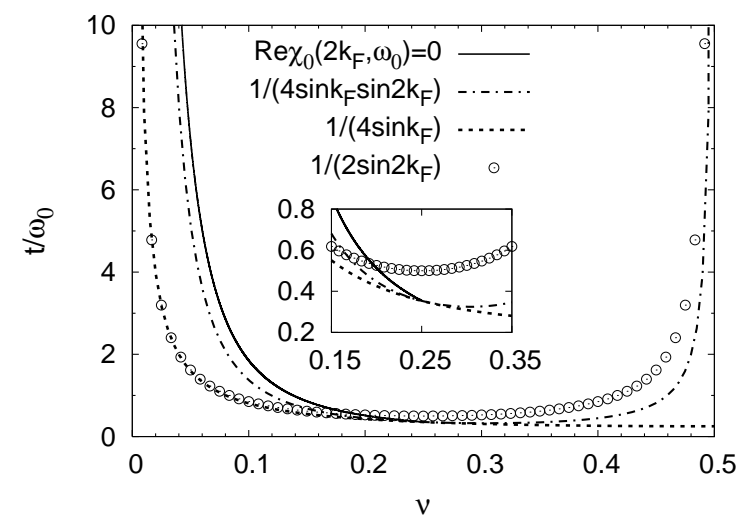

FIG. 3. Curves relevant for identifying different perturbative regimes. Plot of $t / \omega_{0}$ and filling factor $\nu=k_{F} / \pi$ values satisfying $\operatorname{Re} \chi_{0}\left(2 k_{F}, \omega_{0}\right)=0$ and curves for the functions $t / \omega_{0}=1 /\left[4 \sin \left(k_{F}\right) \sin \left(2 k_{F}\right)\right],=1 /\left[4 \sin \left(k_{F}\right)\right]$, and $=1 /\left[2 \sin \left(2 k_{F}\right)\right]$.

It should be pointed out that only the region $\operatorname{Re} \chi_{0}\left(\vec{p}, \omega_{0}\right)<0$ is relevant in obtaining $g_{c}$. The curve $\operatorname{Re} \chi_{0}\left(\vec{p}, \omega_{0}\right)=0$ is depicted in Fig. 3 and exists only for $k_{F}<\pi / 4$. Above (below) this curve, $\operatorname{Re} \chi_{0}\left(\vec{p}, \omega_{0}\right)$ is always negative (positive). Furthermore, for $k_{F}>\pi / 4$ and all values of the adiabaticity parameter $t / \omega_{0}$ above (below) the line $t / \omega_{0}=1 /\left[4 \sin \left(k_{F}\right)\right]$, one can show analytically that $\operatorname{Re} \chi_{0}\left(\vec{p}, \omega_{0}\right)$ is always negative (positive).

It can be shown that, for a given filling $\nu$ and any value of $t / \omega_{0}$ where $\operatorname{Re} \chi_{0}\left(\vec{p}, \omega_{0}\right)<0$, the macroscopic instability condition is always given by Eq. (12) (see Appendix C). For filling factors above 0.25 , as shown in Fig. 2, the $g_{c}$ decreases with decreasing $t / \omega_{0}$ with a downward kink appearing at a certain value of $t / \omega_{0}$ corresponding to the negative divergence of $\chi_{0}\left(\vec{p}, \omega_{0}\right)$. At the point of divergence of $\chi_{0}\left(\vec{p}, \omega_{0}\right)$, perturbation theory is no longer valid. For $k_{F}<\pi / 4$, the $g_{c}$ initially decreases with decreasing $t / \omega_{0}$ until a certain value of $t / \omega_{0}$; while below this value $t / \omega_{0}$, the value of $g_{c}$ again increases due to the fact that $\operatorname{Re} \chi_{0}\left(\vec{p}, \omega_{0}\right)$ value approaches zero value (see Fig. 3). Lastly, we would like to point out that the $g_{c}$ values are not reliable when $g_{c} \omega_{0} / t>1$ and hence in the entire anti-adiabatic regime $\left(t / \omega_{0}<1\right)$ the $g_{c}$ values are suspect [see Fig. 2(b)].

\section{T=0 PHASE DIAGRAM AT NON-HALF-FILLING}

At non-half filling, we will now discuss the quantum phase transition based on the perturbation theory results derived above and the work reported in Ref. [21]. In the extreme small polaron regime, for $g>1$, it was shown earlier that the effective Holstein Hamiltonian can be recast as an effective spin Hamiltonian (using WignerJordan transformation) as follows (see [21] for details): 


$$
\begin{aligned}
H_{e}^{s p i n} \sim-g^{2} \omega_{0} & {\left[\sum_{j} \sigma_{j}^{z}+\zeta e^{-g^{2}} \sum_{j}\left(\sigma_{j}^{+} \sigma_{j+1}^{-}+\text {H.c. }\right)\right.} \\
& -\zeta^{2} \sum_{j} \sigma_{j}^{z} \sigma_{j+1}^{z} \\
& \left.+\zeta^{2} e^{-g^{2}} \sum_{j}\left\{\sigma_{j-1}^{+} \sigma_{j+1}^{-}+\text {H.c. }\right\}\right],
\end{aligned}
$$

where $\zeta \equiv t / g^{2} \omega_{0}$ is the polaron size parameter. The above equation was obtained by assuming that the phonons are frozen in the Lang-Firsov transformed (LFT) phononic ground state $\exp \left[g \Sigma_{j} c_{j}^{\dagger} c_{j}\left(a_{j}-\right.\right.$ $\left.\left.a_{j+1}\right)\right]|0\rangle_{p h}[25]$. Now, for the above Eq. (16) to be the basis for studying phase transition, each of the coefficients of the second, third, and fourth terms on the rhs should be significantly smaller than $\omega_{0}$ (so that the LFT phononic ground state remains unaffected). In Eq. (16), for $\zeta<<1$, the coefficients of the nearest neighbor and the next to nearest neighbor interactions in the transverse direction are much smaller than $\omega_{0}$. Contrastingly, the coefficient of the nearest neighbor interaction in the longitudinal direction is much smaller than $\omega_{0}$ always when $t / \omega_{0}<1$; while for $t / \omega_{0}>1$, it is much smaller only when $\left[t /\left(g \omega_{0}\right)\right]^{2}<<1$ (i.e., for large values of $g$ ). Note that, when $\zeta<<1$, the last term is negligible. Using Bethe ansatz, we know that anisotropic Heisenberg model always yields a Luttinger liquid away from halffilling [26-28]! From the above analysis, it follows that a LL results for all values of $1 / \zeta>>\max \left(1, t / \omega_{0}\right)$. Furthermore, for $t / \omega_{0}<<1$ and $g>1$, the above Eq. (16) is valid with the last term on the rhs being negligible and consequently LL results away from half-filling.

Next, when $g<1$ and $t / \omega_{0}<<1$, we get the corresponding effective spin Hamiltonian from the effective Holstein Hamiltonian to be [21]

$$
\begin{aligned}
H_{e}^{s p i n} \sim-g^{2} \omega_{0} & {\left[\sum_{j} \sigma_{j}^{z}+\zeta e^{-g^{2}} \sum_{j}\left(\sigma_{j}^{+} \sigma_{j+1}^{-}+\text {H.c. }\right)\right.} \\
& +\left(\frac{t}{\omega_{0}}\right)^{2} e^{-2 g^{2}} \sum_{j}\left\{\sigma_{j-1}^{+} \sigma_{j+1}^{-}+\text {H.c. }\right\} \\
& \left.-4\left(\frac{t}{\omega_{0}}\right)^{2} e^{-2 g^{2}} \sum_{j} \sigma_{j}^{z} \sigma_{j+1}^{z}\right]
\end{aligned}
$$

In arriving at the above equation too, it was assumed that the phonons are in the LFT phononic ground state. Such an assumption is justified because the coefficients of the second, third, and fourth terms on the rhs of the above equation, are much smaller than $\omega_{0}$. In Eq. (17), the small parameter is $t / \omega_{0}$ and the last two terms are negligible compared to the second term when the adiabaticity parameter $t / \omega_{0}<<1$. Then, this implies a LL state for all fillings. For $g \rightarrow 0$ and any value of $t / \omega_{0}$, we do not expect a CDW state.

The phase diagram (see Fig. 4) is drawn qualitatively for a general filling away from half-filling. For $\nu<0.25$, the CDW region shifts to the right with decreasing $\nu$ as can be surmised from the region of validity $g_{c} \omega_{0} / t<1$ in Fig. 2(b). The regions where LL is certain is indicated. For $t / \omega_{0}>1$, since we need $\left[t /\left(g \omega_{0}\right)\right]^{2}<<1$ for the validity of Eq. (16), the boundary of the LL-certain-region is linear and of the form $g^{2} \omega_{0} / t=D t / \omega_{0}$ where the slope $D>>1$. Furthermore, for $g \omega_{0} / t<<1$, one expects a LL phase and hence we get a linear boundary (of the form $g^{2} \omega_{0} / t=d t / \omega_{0}$ with $d<<1$ ) for the LL phase in the lower left part of the diagram.

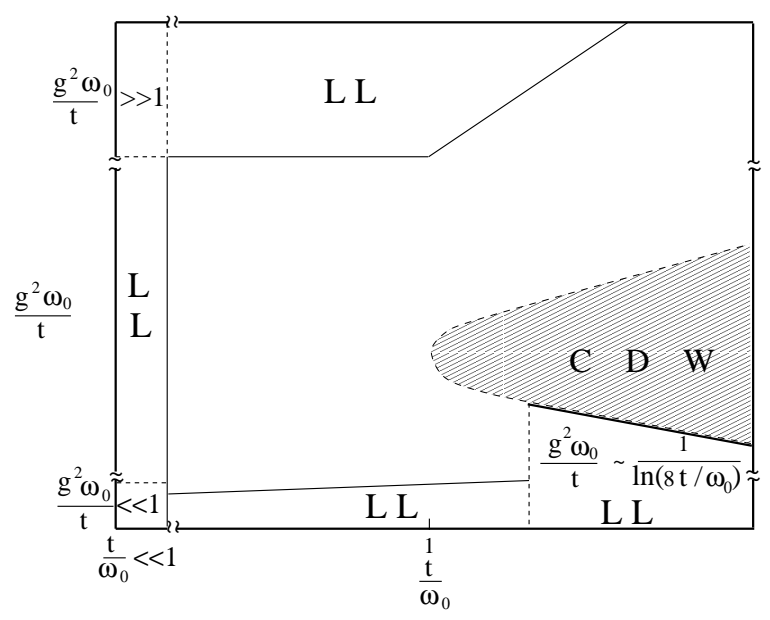

FIG. 4. Zero temperature phase diagram at non-half filling. Regions where CDW and LL phases certainly exist are depicted. The calculated transition from LL to CDW is indicated by a dark line along with its expression.

The thatched portion corresponds to a region where a CDW state is certain. The upper boundary of the thatched region, which is obtained by the condition $g \omega_{0} / t \sim 1$ and is therefore linear with slope of order unity, corresponds to the breakdown of the perturbation theory used in identifying the CDW transition. The only certain location of the transition from LL to CDW is indicated by a solid dark line and is approximately given by $g^{2} \omega_{0} / t \sim 1 /\left(\ln \left(8 t / \omega_{0}\right)\right.$ [while the exact relation is expressed in Eq. (14)]. However, it is unclear in the rest of the figure where exactly the transition from a CDW state to a LL state takes place.

Finally, it should be emphasized that there are two different types of phase transitions. The nature of the phase transition on the adiabatic side, at intermediate values of $g<t / \omega_{0}$, is driven by a macroscopic ionic lattice distortion. Quite differently, in the restricted region of the small polaron limit where $1 / \zeta>>\max \left(1, t / \omega_{0}\right)$ and in the extreme anti-adiabatic regime $\left(t / \omega_{0}<<1\right)$, the $\mathrm{CDW}$ is driven by a small-polaron-interaction based 
mechanism. In the latter case, the coordinate Bethe ansatz argument precludes the possibility of a small polaronic CDW away from half-filling in sharp contrast to the half-filled case $[19,21]$.

\section{ACKNOWLEDGEMENTS}

One of the authors (S.Y.) would like to thank G. Baskaran and S. M. Bhattacharjee for useful discussions. This work was partially funded by CAMACS of SINP.

[1] For a review see Polarons and Bipolarons, by Alexandrov, A. S. \& Mott, N. (World Scientific, Singapore, 1995).

[2] Holstein, T., Studies of polaron motion. Part II. The "small" polaron. Ann. Phys. (N.Y.) 8, 343 (1959).

[3] For a recent review see Fehske, H. \& Jeckelmann, E. Quantum phase transitions in one-dimensional electronphonon systems. cond-mat/0510741.

[4] Peierls, R. E. Quantum Theory of Solids (Oxford University Press, New York, 1955).

[5] Ishiguro, T., Yamaji, K., \& Saito, G. Organic Superconductors (Springer, Berlin, 1998).

[6] Tsuda, N., Nasu, K., Yanase, A., \& Siratori, K. Electronic Conduction in Oxides (Springer-Verlag, Berlin 1990).

[7] Monceau, P. Electronic Properties of Inorganic QuasiOne-Dimensional Compounds, Part I, (D. Reidel Publishing Company, Dordrecht, Holland, 1985).

[8] Grüner, G. Density Waves in Solids, (Addison-Wesley, Reading, MA, 1994).

[9] For a review see Colossal Magnetoresistance, Charge Ordering, and Related Properties of Manganese Oxides, edited by Rao, C. N. R. and Raveau, B. (World Scientific, Singapore, 1998).

[10] Datta, S. and Yarlagadda, S. Quantum phase transition in the Holstein model. Proceedings of the DAE Solid State Symposium (Golden Jubilee) 50, 605 (2005).

[11] Pai, G. V., Hassan, S. R., Krishnamurthy, H. R., \& Ramakrishnan, T. V. Zero temperature insulator-metal transition in doped manganites. Europhys. Lett. 64, 696 (2003).

[12] Hirsch, J. E., \& Fradkin, E. Effect of quantum fluctuations on the Peierls instability: a Monte Carlo study. Phys. Rev. Lett. 49, 402 (1982).
[13] Hirsch, J. E., \& Fradkin, E. Phase diagram of onedimensional electron-phonon systems. II. The molecular crystal model. Phys. Rev. B 27, 4302 (1983).

[14] McKenzie, R. H., Hamer, C. J., \& Murray, D. W. Quantum Monte Carlo study of the one-dimensional Holstein model of spinless fermions. Phys. Rev. B 53, 9676 (1996).

[15] Creffield, C. E., Sangiovanni, G. \& Capone, M. Phonon softening and dispersion in the 1D Holstein model of spinless fermions. cond-mat/0502387.

[16] Caron, L. G., \& Bourbonnais, C. Two-cutoff renormalization and quantum versus classical aspects for the onedimensional electron-phonon system. Phys. Rev. B 29, 4230 (1984).

[17] Zheng, H., Feinberg, D., \& Avignon, M. Effect of quantum fluctuations on the Peierls dimerization in the onedimensional molecular-crystal model. Phys. Rev. B 39, 9405 (1989).

[18] Perroni, C. A. et al. Infrared conductivity of a onedimensional charge-ordered state: Quantum lattice effects. Phys. Rev. B 67, 214301 (2003).

[19] Bursill, R. J., McKenzie, R. H., \& Hamer, C. J. Phase diagram of the one-dimensional Holstein model of spinless fermions. Phys. Rev. Lett. 80, 5607 (1998).

[20] Fehske, H., Holicki, M., \& Weiße, A. Lattice dynamical effects on the Peierls transition in one-dimensional metals and spin chains. Advances in solid state physics $\mathbf{4 0}$, 235 (2000).

[21] Datta, S., Das, A., \& Yarlagadda, S. Many-polaron effects in the Holstein model. Phys. Rev. B 71, 235118 (2005).

[22] We are only aware of the numerical work of Hohenadler, M. et al. Photoemission spectra of many-polaron systems. Phys. Rev. B 71, 245111 (2005).

[23] Lindhard, J. On the properties of a gas of charged particles. Kgl. Danske Videnskab. Selsk. Mat. Fys. Medd. 28(8), 1 (1954).

[24] Due to inversion symmetry $\chi_{0}\left(\vec{p}, \omega_{0}\right)=\chi_{0}\left(-\vec{p}, \omega_{0}\right)$.

[25] Lang, I. G., \& Firsov, Yu. A. Kinetic theory of semiconductors with low mobility. Zh. Eksp. Teor. Fiz. 43, 1843 (1962) [Sov. Phys. JETP 16, 1301 (1962)].

[26] For a review see Bose, I. in Field Theories in Condensed Matter Physics, edited by Sumathi Rao (Institute of Physics Publishing, Bristol, 2002).

[27] For a review see Quantum Physics in One Dimension, by Giamarchi, T. (Oxford University Press, New York, 2004).

[28] Haldane, F. D. M. General relation of correlation exponents and spectral properties of one-dimensional Fermi systems: Applications to the anisotropic $\mathrm{S}=1 / 2$ Heisenberg chain. Phys. Rev. Lett. 45, 1358 (1980). 


\section{APPENDIX A}

The interacting state $\left|\Psi_{n}^{0}\right\rangle$, corresponding to the non-interacting state $\left|\psi_{n}^{0}\right\rangle=\left|0 ; n_{-\vec{p}}\right\rangle$, yields the following energy expression:

$$
E_{\Psi_{n}^{0}}=T_{0}+n \omega_{0}-\sum_{\phi_{l} \neq \psi_{n}^{0}}\left[\frac{\left|\left\langle\phi_{l}\left|H_{1}\right| \psi_{n}^{0}\right\rangle\right|^{2}}{E_{\phi_{l}}^{0}-E_{\psi_{n}^{0}}^{0}}\right]
$$

where $T_{0}=E_{\phi_{0}}^{0}=-2 N t \sin \left(k_{F}\right) / \pi$ is the non-interacting kinetic energy of the ground state $\left|\phi_{0}\right\rangle$ and

$$
\begin{aligned}
\sum_{\phi_{l} \neq \psi_{n}^{0}} \frac{\left|\left\langle\phi_{l}\left|H_{1}\right| \psi_{n}^{0}\right\rangle\right|^{2}}{E_{\phi_{l}}^{0}-E_{\psi_{n}^{0}}^{0}}=g^{2} \omega_{0}^{2} & {\left[\frac{1}{N} \sum_{m \neq 0} \sum_{\vec{q} \neq \vec{p}} \frac{\left|\left\langle\left. m\right|_{e l} \rho_{\vec{q}} \mid 0\right\rangle_{e l}\right|^{2}}{\xi_{m 0}+\omega_{0}}+\frac{n+1}{N} \sum_{m \neq 0} \frac{\left|\left\langle\left. m\right|_{e l} \rho_{\vec{p} \mid} \mid 0\right\rangle_{e l}\right|^{2}}{\xi_{m 0}+\omega_{0}}\right.} \\
+ & \left.\frac{n}{N} \sum_{m \neq 0} \frac{\left|\left\langle\left. m\right|_{e l} \rho_{-\vec{p}} \mid 0\right\rangle_{e l}\right|^{2}}{\xi_{m 0}-\omega_{0}-i \eta}\right] \\
=g^{2} \omega_{0}^{2} & {\left[\frac{1}{N} \sum_{\vec{q}, m \neq 0} \frac{\left|\left\langle\left. m\right|_{e l} \rho_{\vec{q}} \mid 0\right\rangle_{e l}\right|^{2}}{\xi_{m 0}+\omega_{0}}-n \chi_{0}\left(\vec{p}, \omega_{0}\right)\right] }
\end{aligned}
$$

with $\eta \rightarrow 0^{+}$. Then the above Eq. (A2) yields the energy difference $E_{\Psi_{n+1}^{0}}-E_{\Psi_{n}^{0}}=\omega_{0}+\Sigma\left(-\vec{p}, \omega_{0}\right)$ where the self-energy of a phonon $\Sigma\left(\vec{p}, \omega_{0}\right)=g^{2} \omega_{0}^{2} \chi_{0}\left(\vec{p}, \omega_{0}\right)$. The self-energy is displayed in Fig. 5 with the bubble representing the polarizability $\chi_{0}\left(\vec{p}, \omega_{0}\right)$ and each of the electron-phonon interaction vertices corresponding to the factor $g \omega_{0}$.

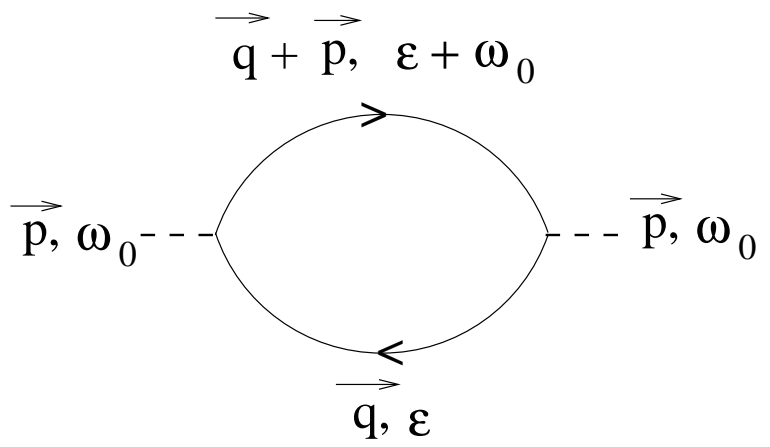

FIG. 5. Self-energy of a phonon. The solid and the dashed lines depict the electron and the phonon propagators respectively.

\section{APPENDIX B}

1. The set of degenerate states for half-filled case

The solutions of the expression

$$
\omega_{0}=\epsilon_{\vec{k}-\vec{p}}-\epsilon_{\vec{k}}=4 t \sin \left(k_{F}-k\right) \sin \left(k_{F}\right),
$$

for $p=2 k_{F}$ are given by

$$
k=k_{F}-\arcsin \left(\frac{\omega_{0}}{4 t \sin \left(k_{F}\right)}\right),
$$

and

$$
k=-\pi+k_{F}+\arcsin \left(\frac{\omega_{0}}{4 t \sin \left(k_{F}\right)}\right) .
$$


Then, the set of states that are degenerate with $\left|0 ; n_{-\vec{p}}\right\rangle$ and that should be considered in degenerate perturbation theory consists of the following states $\left|0 ;(n-2 m)_{-\vec{p}} m_{2 \vec{k}-\vec{p}} m_{-2 \vec{k}-\vec{p}}\right\rangle,\left|\vec{k} \rightarrow \vec{k}-\vec{p} ;(n-2 m-1)_{-\vec{p}} m_{2 \vec{k}-\vec{p}} m_{-2 \vec{k}-\vec{p}}\right\rangle$, $\left|-\vec{k} \rightarrow-\vec{k}-\vec{p} ;(n-2 m-1)_{-\vec{p}} m_{2 \vec{k}-\vec{p}} m_{-2 \vec{k}-\vec{p}}\right\rangle,\left|\vec{k} \rightarrow \vec{k}-\vec{p},-\vec{k} \rightarrow-\vec{k}-\vec{p} ;(n-2 m-2)_{-\vec{p}} m_{2 \vec{k}-\vec{p}} m_{-2 \vec{k}-\vec{p}}\right\rangle, \mid \vec{k} \rightarrow$ $\left.-\vec{k}-\vec{p} ;(n-2 m-2)_{-\vec{p}}(m+1)_{2 \vec{k}-\vec{p}} m_{-2 \vec{k}-\vec{p}}\right\rangle,\left|-\vec{k} \rightarrow \vec{k}-\vec{p} ;(n-2 m-2)_{-\vec{p}} m_{2 \vec{k}-\vec{p}}(m+1){ }_{-2 \vec{k}-\vec{p}}\right\rangle$, where $\mathrm{m}=0,1,2,3, \ldots$ with the constraint that the number of phonons is non-negative. Thus we see that the number of degenerate states increases linearly with $n$ and is given by $3 n$.

\section{Derivation of energy $E_{\Phi_{n}}$ in the half-filled case}

The starting state $\left|\phi_{n}\right\rangle \equiv\left|-\overrightarrow{k_{F}} \rightarrow \vec{k}-\vec{p}, \overrightarrow{k_{F}} \rightarrow-\vec{k}-\vec{p} ; n_{-\vec{p}}\right\rangle(n \geq 1)$, after switching on the interactions, results in the following energy:

$$
E_{\Phi_{n}}=T_{0}+(n+1) \omega_{0}-\sum_{l \neq n}\left[\frac{\left|\left\langle\phi_{l}\left|H_{1}\right| \phi_{n}\right\rangle\right|^{2}}{E_{\phi_{l}}^{0}-E_{\phi_{n}}^{0}}\right]
$$

To evaluate the last term on the right hand side (rhs) of Eq. (B4), we use Eq. (A2) and obtain

$$
\begin{array}{r}
\sum_{l \neq n} \frac{\left|\left\langle\phi_{l}\left|H_{1}\right| \phi_{n}\right\rangle\right|^{2}}{E_{\phi_{l}}^{0}-E_{\phi_{n}}^{0}}-\operatorname{Re} \sum_{\phi_{l} \neq \psi_{n}^{0}} \frac{\left|\left\langle\phi_{l}\left|H_{1}\right| \psi_{n}^{0}\right\rangle\right|^{2}}{E_{\phi_{l}}^{0}-E_{\psi_{n}^{0}}^{0}}=-g^{2} \omega_{0}^{2} \operatorname{Re}\left[\Pi_{0}\left(\vec{k}-\vec{p},-\vec{k}_{F}, \omega_{0}\right)\right. \\
\left.+\Pi_{0}\left(-\vec{k}-\vec{p}, \vec{k}_{F}, \omega_{0}\right)\right] \\
=-2 g^{2} \omega_{0}^{2} \operatorname{Re}_{0}\left(\vec{k}-\vec{p}, \vec{k}_{F}, \omega_{0}\right)
\end{array}
$$

where

$$
\begin{aligned}
\operatorname{Re}_{0}\left(\vec{s}, \vec{q}, \omega_{0}\right) \equiv-\frac{1}{N} \sum_{\vec{r}}[ & \frac{1-n_{\vec{r}}}{\epsilon_{\vec{r}}+\omega_{0}-\epsilon_{\vec{s}}}+\frac{n_{\vec{r}}}{\epsilon_{\vec{q}}+\omega_{0}-\epsilon_{\vec{r}}} \\
& \left.-\frac{n_{\vec{r}}}{\epsilon_{\vec{s}}+\omega_{0}-\epsilon_{\vec{r}}}-\frac{1-n_{\vec{r}}}{\epsilon_{\vec{r}}+\omega_{0}-\epsilon_{\vec{q}}}\right] .
\end{aligned}
$$

In the above expression for $\Pi_{0}$, the first and second terms correspond to adding the contributions due to the electron at $\vec{s}$ going to a state outside the Fermi surface (FS) and those due to the electrons within the FS going to the state $\vec{q}$ respectively. Whereas the third and fourth terms, on the rhs of Eq. (B6), represent subtracting contributions due to electrons within the FS going to the state $\vec{s}$ and those due to electron at $\vec{q}$ going outside the FS respectively. In obtaining Eq. (B5), the terms that are ignored or overcounted are negligible for large $N$. From the main text we know that the state $\left|\phi_{0}\right\rangle$, upon turning on the interaction, yields the energy

$$
E_{\Phi_{0}}=T_{0}-\frac{g^{2} \omega_{0}^{2}}{N} \sum_{\vec{q}, m \neq 0} \frac{\left|\left\langle\left. m\right|_{e l} \rho_{\vec{q}} \mid 0\right\rangle_{e l}\right|^{2}}{\xi_{m 0}+\omega_{0}} .
$$

Then, from Eqs. (B4)-(B7), we obtain

$$
\begin{aligned}
E_{\Phi_{n}}= & E_{\Phi_{0}}+(n+1) \omega_{0}\left[1+g^{2} \omega_{0} \operatorname{Re} \chi_{0}\left(\vec{p}, \omega_{0}\right)\right] \\
& +g^{2} \omega_{0}^{2} \operatorname{Re}\left[2 \Pi_{0}\left(\vec{k}-\vec{p}, \vec{k}_{F}, \omega_{0}\right)-\chi_{0}\left(\vec{p}, \omega_{0}\right)\right]
\end{aligned}
$$

In the above equation, $2 \Pi_{0}-\chi_{0}>0$ for all values of $t / \omega_{0}>1$. 


\section{APPENDIX C}
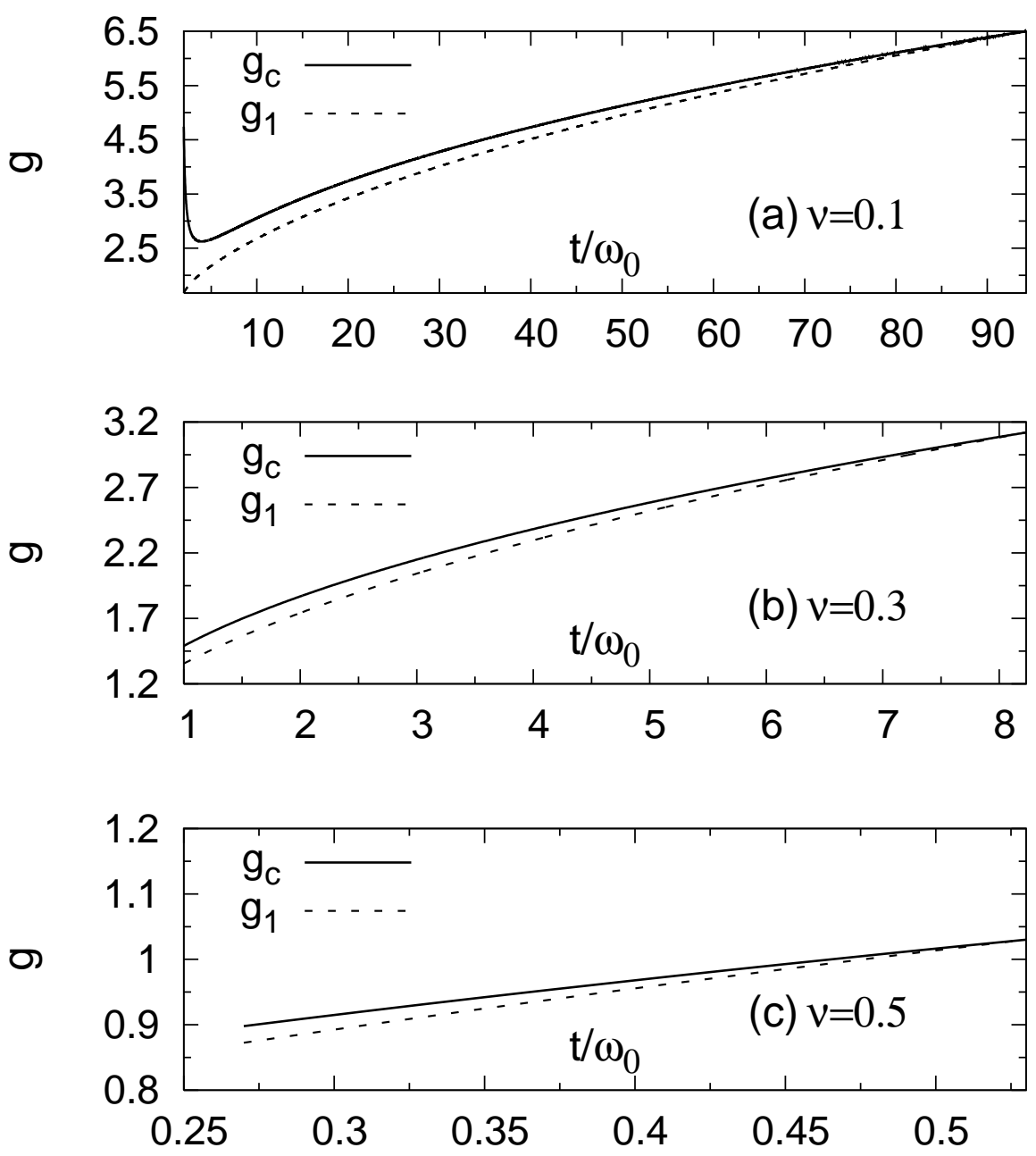

FIG. 6. Plots of $g_{c}$ and $g_{1}$ (drawn till their crossing point) versus $t / \omega_{0}$ when the excitation expression $\epsilon_{\vec{k}-\vec{p}}-\epsilon_{\vec{k}}=\omega_{0}$ has only one solution $\vec{k}$ (with $k<k_{F}=p / 2$ ) for (a) $\nu=0.1$ and (b) $\nu=0.3$ and two solutions for (c) $\nu=0.5$.

Here, we consider in detail the non-half-filled case. For $k_{F}$ larger (smaller) than $\pi / 4$, the line given by $t / \omega_{0}=$ $1 /\left[4 \sin \left(2 k_{F}\right) \sin \left(k_{F}\right)\right]$ corresponds to the larger (smaller) magnitude wavevector solution $\vec{k}$ lying on the Fermi surface as seen from Eq. (B3) (Eq. (B2)). In the region above (below) $t / \omega_{0}=1 /\left[4 \sin \left(2 k_{F}\right) \sin \left(k_{F}\right)\right]$ (see Fig. 3 in the main text), the excitation energy expression $\epsilon_{\vec{k}-\vec{p}}-\epsilon_{\vec{k}}=\omega_{0}$ with $p=2 k_{F}$ is satisfied by one (two) wavevector(s) for $|k|<k_{F}>\pi / 4$ [as seen from Eq. (B3)] whereas for $|k|<k_{F}<\pi / 4$ it is satisfied by one (zero) wavevector(s) [as seen from Eq. (B2)].

In the region $t / \omega_{0}>1 /\left[4 \sin \left(2 k_{F}\right) \sin \left(k_{F}\right)\right]$, the degenerate states are $\left|\psi_{n}^{0}\right\rangle \equiv\left|0 ; n_{-\vec{p}}\right\rangle$ and $\left|\psi_{n}^{1}\right\rangle \equiv \mid \vec{k} \rightarrow \vec{k}-\vec{p} ;(n-$ $\left.1)_{-\vec{p}}\right\rangle$. Unlike at half-filling, only two (i.e., the above given two) degenerate states need be considered for carrying out degenerate perturbation theory. The two basis states are $\left|\psi_{n}^{-}\right\rangle \equiv\left[\left|\psi_{n}^{0}\right\rangle-\left|\psi_{n}^{1}\right\rangle\right] / \sqrt{2}$ and $\left|\psi_{n}^{+}\right\rangle \equiv\left[\left|\psi_{n}^{0}\right\rangle+\left|\psi_{n}^{1}\right\rangle\right] / \sqrt{2}$ with $\left|\psi_{n}^{-}\right\rangle\left(\left|\psi_{n}^{+}\right\rangle\right)$yielding the lower (higher) eigen energy. Then, from degenerate perturbation theory, one gets

$$
E_{\Psi_{n}^{-}}=E_{\psi_{n}^{-}}-\frac{\sqrt{n}}{\sqrt{N}} g \omega_{0}-\sum_{\phi_{l} \neq \psi_{n}^{-}, \psi_{n}^{+}} \frac{\left|\left\langle\phi_{l}\left|H_{1}\right| \psi_{n}^{-}\right\rangle\right|^{2}}{E_{\phi_{l}}^{0}-E_{\psi_{n}^{-}}^{0}}
$$

where the second term on the rhs is the first order energy correction; furthermore, it is understood that the states $\left|\phi_{l}\right\rangle$ do not belong to the subspace spanned by $\left|\psi_{n}^{ \pm}\right\rangle$. Next, to evaluate the last term on the rhs of the above Eq. (C1), we 
use the following non-mixing fact:

$$
\sum_{\phi_{l} \neq \psi_{n}^{-}, \psi_{n}^{+}} \frac{\left|\left\langle\phi_{l}\left|H_{1}\right| \psi_{n}^{-}\right\rangle\right|^{2}}{E_{\phi_{l}}^{0}-E_{\psi_{n}^{-}}^{0}}=\frac{1}{2} \sum_{\phi_{l} \neq \psi_{n}^{-}, \psi_{n}^{+}} \frac{\left|\left\langle\phi_{l}\left|H_{1}\right| \psi_{n}^{0}\right\rangle\right|^{2}}{E_{\phi_{l}}^{0}-E_{\psi_{n}^{0}}^{0}}+\frac{1}{2} \sum_{\phi_{l} \neq \psi_{n}^{-}, \psi_{n}^{+}} \frac{\left|\left\langle\phi_{l}\left|H_{1}\right| \psi_{n}^{1}\right\rangle\right|^{2}}{E_{\phi_{l}}^{0}-E_{\psi_{n}^{1}}^{0}} .
$$

Using Eq. (A2) and on noting that

$$
\begin{aligned}
\sum_{\phi_{l} \neq \psi_{n}^{-}, \psi_{n}^{+}} \frac{\left|\left\langle\phi_{l}\left|H_{1}\right| \psi_{n}^{1}\right\rangle\right|^{2}}{E_{\phi_{l}}^{0}-E_{\psi_{n}^{1}}^{0}}=g^{2} \omega_{0}^{2} & {\left[\frac{1}{N} \sum_{\vec{q}, m \neq 0} \frac{\left|\left\langle\left. m\right|_{e l} \rho_{\vec{q}} \mid 0\right\rangle_{e l}\right|^{2}}{\xi_{m 0}+\omega_{0}}\right.} \\
& \left.-(n-1) \operatorname{Re} \chi_{0}\left(\vec{p}, \omega_{0}\right)-\operatorname{Re} \Pi_{0}\left(\vec{k}-\vec{p}, \vec{k}, \omega_{0}\right)\right],
\end{aligned}
$$

we obtain from Eqs. (C1)-(C3)

$$
\begin{aligned}
E_{\Psi_{n}^{-}}= & E_{\Phi_{0}}+E^{1}+n \omega_{0}\left[1+g^{2} \omega_{0} \operatorname{Re} \chi_{0}\left(\vec{p}, \omega_{0}\right)\right] \\
& -0.5 g^{2} \omega_{0}^{2} \operatorname{Re}\left[\chi_{0}\left(\vec{p}, \omega_{0}\right)-\Pi_{0}\left(\vec{k}-\vec{p}, \vec{k}, \omega_{0}\right)\right] .
\end{aligned}
$$

In Eq. (C3), $\Pi_{0}\left(\vec{k}-\vec{p}, \vec{k}, \omega_{0}\right)$ has been obtained in a manner similar to that in the half filled case. As mentioned in the main text, the macroscopic instability condition is given by

$$
1+2 g_{c}^{2} \omega_{0} \operatorname{Re} \chi_{0}\left(\vec{p}, \omega_{0}\right)=0 .
$$

However, for each filling below half-filling, there is a corresponding critical value of the adiabaticity parameter $\left(t / \omega_{0}\right)$ below which $\operatorname{Re}\left[\chi_{0}-\Pi_{0}\right]>0$ in the above Eq. (C5). Consequently, the interacting state $\left|\Psi_{1}^{-}\right\rangle$produced by the one phonon state $\left|\psi_{1}^{-}\right\rangle$is more stable than the interacting state $\left|\Phi_{0}\right\rangle$ at an electron-phonon coupling value given by $g_{1}<g<g_{c}$ where $g_{1}$ corresponds to $\left(E_{\Psi_{1}^{-}}-E_{\Phi_{0}}\right)^{2}=0$ [see Fig. 6]. Only above this critical adiabaticity parameter value do we have $\left|\Psi_{n}^{-}\right\rangle$, for $n \rightarrow \infty$, as the most stable state at a coupling $g>g_{c}<g_{1}$. Furthermore, it should also be noted that the interacting state $\left|\Psi_{1}^{-}\right\rangle$is also a LL. Thus, it is obvious that the LL to CDW transition occurs only at $g=g_{c}^{+}$. Lastly it should also be mentioned that, instead of using the above degenerate perturbation theory to obtain the instability condition, one can also adopt a blocking approach similar to that at half-filling by exciting one electron at the Fermi surface to the state $\vec{k}-\vec{p}$ and then employ non-degenerate perturbation theory. In the latter case, the condition for macroscopic instability is still the same while the critical adiabaticity parameter value (above which $\left.g_{c}<g_{1}\right)$ is slightly larger.

1. The case when $\epsilon_{\vec{k}-\vec{p}}-\epsilon_{\vec{k}}=\epsilon_{\vec{k}-2 \vec{p}}-\epsilon_{\vec{k}-\vec{p}}=\omega_{0}$

In the non-half-filled regime, we will now consider the special case where the excitation expression $\epsilon_{\vec{k}-\vec{p}}-\epsilon_{\vec{k}}=\omega_{0}$ and $\epsilon_{\vec{k}-2 \vec{p}}-\epsilon_{\vec{k}-\vec{p}}=\omega_{0}$ are simultaneously satisfied by one $\vec{k}$ (with $k<k_{F}=p / 2$ ). This will hold when $2 t \cos (k)=\omega_{0}$ and consequently when $t / \omega_{0}=1 /\left[2 \sin \left(2 k_{F}\right)\right]$. For values of the adiabaticity parameter $t / \omega_{0}$ and $k_{F}$ that lie on the line $t / \omega_{0}=1 /\left[2 \sin \left(2 k_{F}\right)\right]$ depicted in Fig. 3 of main text, the method involving only two degenerate states breaks down. To analyze the CDW instability, one can use a blocking method similar to that used at half-filling in the main text. We Pauli block the states $\vec{k}-\vec{p}$ and $\vec{k}-2 \vec{p}$ by the two electrons on the Fermi surface. The blocked state $\left|\phi_{n}^{\prime}\right\rangle \equiv\left|\overrightarrow{k_{F}} \rightarrow \vec{k}-\vec{p},-\overrightarrow{k_{F}} \rightarrow \vec{k}-2 \vec{p} ; n_{-\vec{p}}\right\rangle$ yields the energy for the interacting state $\left|\Phi_{n}^{\prime}\right\rangle$ to be

$$
\begin{aligned}
E_{\Phi_{n}^{\prime}}= & E_{\Phi_{0}}+\omega_{0}\left(1+\csc \left(k_{F}\right)\right)+n \omega_{0}\left[1+g^{2} \omega_{0} \operatorname{Re} \chi_{0}\left(\vec{p}, \omega_{0}\right)\right] \\
& +g^{2} \omega_{0}^{2} \operatorname{Re}\left[\Pi_{0}\left(\vec{k}-\vec{p}, \vec{k}_{F}, \omega_{0}\right)+\Pi_{0}\left(\vec{k}-2 \vec{p},-\vec{k}_{F}, \omega_{0}\right)\right] .
\end{aligned}
$$

Here too the critical coupling $g_{c}$, for macroscopic instability, is still given by Eq. (C6). However, for filling factors less than approximately 0.22 , the one phonon interacting state $\left|\Phi_{1}^{\prime}\right\rangle$ is the lowest energy state for $g_{1}<g<g_{c}$ (see Fig. 7). At fillings above 0.22 , the large $n$ phonon interacting state $\left|\Phi_{n}^{\prime}\right\rangle$ is the most stable state for $\left.g\right\rangle g_{c}\left\langle g_{1}\right.$. It should also be mentioned that the above blocking procedure can also be used when $\epsilon_{\vec{k}-\vec{p}}-\epsilon_{\vec{k}}=\omega_{0}$ and $\epsilon_{\vec{k}-2 \vec{p}}-\epsilon_{\vec{k}-\vec{p}} \approx \omega_{0}$ in which case the values of $t / \omega_{0}$ and $k_{F}$ lie close to the curve $t / \omega_{0}=1 /\left[2 \sin \left(2 k_{F}\right)\right]$. 


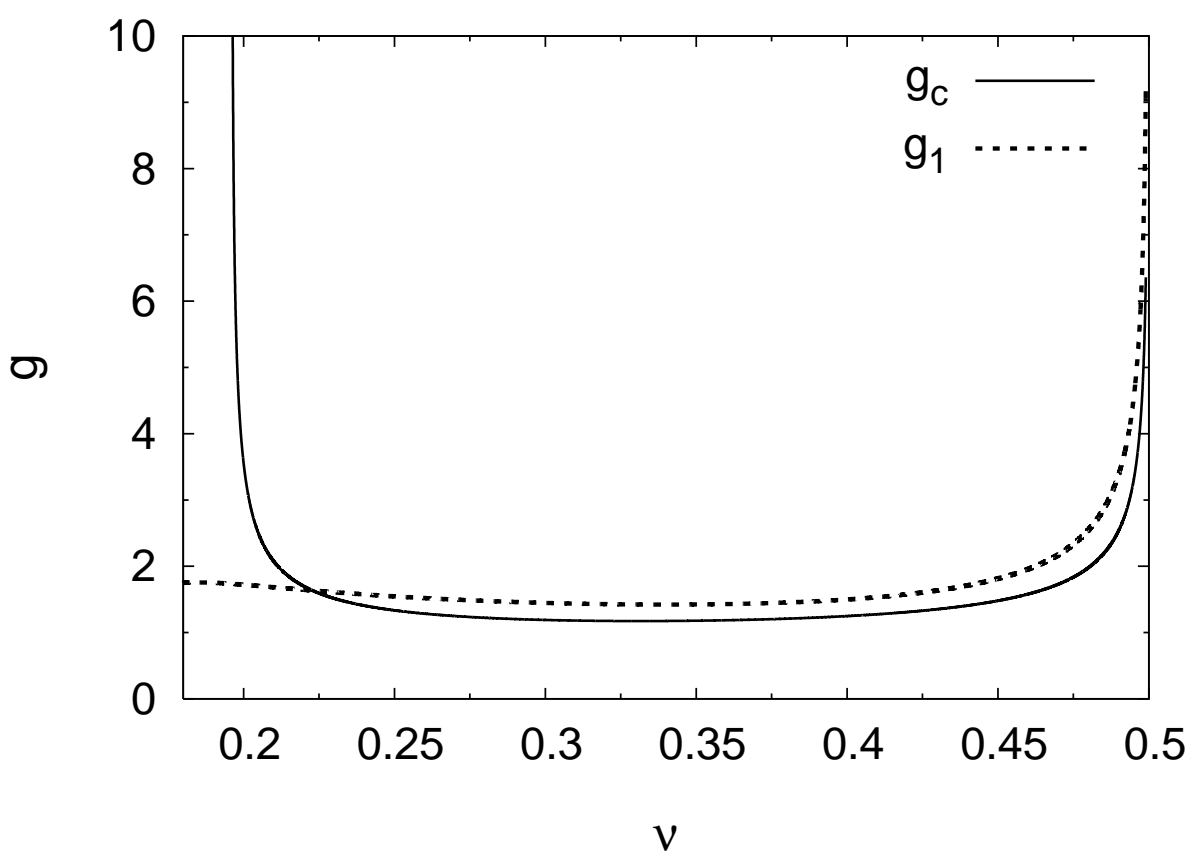

FIG. 7. Plot of $g_{c}$ and $g_{1}$ versus fillings $\nu$ when the excitation expressions $\epsilon_{\vec{k}-\vec{p}}-\epsilon_{\vec{k}}=\omega_{0}$ and $\epsilon_{\vec{k}-2 \vec{p}}-\epsilon_{\vec{k}-\vec{p}}=\omega_{0}$ are simultaneously satisfied by one $\vec{k}$ (with $k<k_{F}=p / 2$ ).

\section{When both the solutions exist}

Here we will consider the lattice instability for the case $t / \omega_{0}<1 /\left[4 \sin \left(2 k_{F}\right) \sin \left(k_{F}\right)\right]$ when two wavevectors satisfy the excitation energy expression $\epsilon_{\vec{k}-\vec{p}}-\epsilon_{\vec{k}}=\omega_{0}$ for $k_{F}>\pi / 4$. For $k_{F}<\pi / 4$, only the region $t / \omega_{0}>1 /\left[4 \sin \left(2 k_{F}\right) \sin \left(k_{F}\right)\right]$ is relevant as $\operatorname{Re} \chi_{0}\left(\vec{p}, \omega_{0}\right)=0$ lies above this line (see Fig. 3 of main text). For $k_{F}>\pi / 4$, let the two wavevectors that satisfy $\epsilon_{\vec{k}-\vec{p}}-\epsilon_{\vec{k}}=\omega_{0}$ be $\vec{k}_{1}$ and $\vec{k}_{2}$. We will use the blocking method to block the states $\vec{k}_{1}-\vec{p}$ and $\vec{k}_{2}-\vec{p}$ by the two electrons on the Fermi surface. The state $\left|\phi_{n}^{\prime \prime}\right\rangle \equiv\left|\overrightarrow{k_{F}} \rightarrow \overrightarrow{k_{1}}-\vec{p},-\overrightarrow{k_{F}} \rightarrow \overrightarrow{k_{2}}-\vec{p} ; n_{-\vec{p}}\right\rangle$ leads to the energy

$$
\begin{aligned}
E_{\Phi_{n}^{\prime \prime}}= & E_{\Phi_{0}}+\omega_{0}+4 t \cos \left(k_{F}\right)+n \omega_{0}\left[1+g^{2} \omega_{0} \operatorname{Re} \chi_{0}\left(\vec{p}, \omega_{0}\right)\right] \\
& +g^{2} \omega_{0}^{2} \operatorname{Re}\left[\Pi_{0}\left(\vec{k}_{1}-\vec{p}, \vec{k}_{F}, \omega_{0}\right)+\Pi_{0}\left(\vec{k}_{2}-\vec{p},-\vec{k}_{F}, \omega_{0}\right)\right] .
\end{aligned}
$$

Here also the macroscopic instability occurs when $g>g_{c}$ with $g_{c}$ obtained from Eq. (C6). For extreme values of $k_{F}$, i.e., $k_{F}$ close to $\pi / 4$ or $\pi / 2$, the large $n$ state $\left|\Phi_{n}^{\prime \prime}\right\rangle$ is the lowest energy state for $g>g_{c}<g_{1}$ (see Fig. 8). On the other hand, at intermediate values of $k_{F}$, the state $\left|\Phi_{1}^{\prime \prime}\right\rangle$ is the stable state for $g_{1}<g<g_{c}$ (see Fig. 8). 

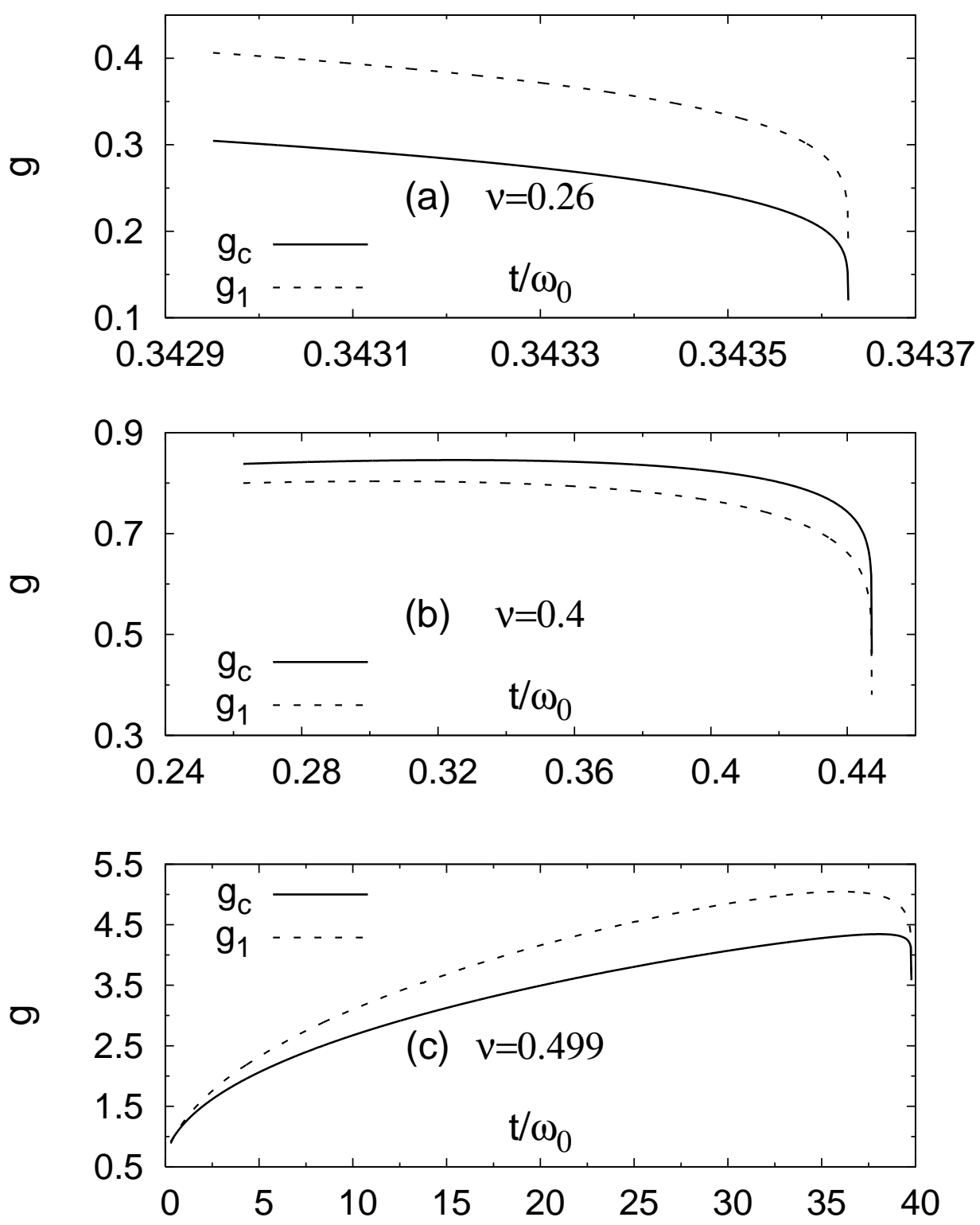

FIG. 8. Plots of $g_{c}$ and $g_{1}$ versus $t / \omega_{0}$ when the excitation expression $\epsilon_{\vec{k}-\vec{p}}-\epsilon_{\vec{k}}=\omega_{0}$ has two solutions $\vec{k}$ (with $k<k_{F}=p / 2$ ) for (a) $\nu=0.26$, (b) $\nu=0.4$, and (c) $\nu=0.499$. 